\title{
LA GESTIÓN DE LAS UNIVERSIDADES DEL CONSEJO DE RECTORES
}

\section{Introducción}

El contexto en el que se desenvuelve la educación superior está marcado en la actualidad por un conjunto de rasgos que representan cambios profundos y probablemente irreversibles. En general, hay un apreciable grado de consenso entre analistas, autoridades y actores respecto de que, en el mundo actual, la educación superior se caracteriza por una considerable masificación, manifestada en la expansión de la matrícula; una creciente diversificación de las instituciones, niveles de formación y programas académicos y profesionales; una generalizada reducción del gasto público sumada a una sostenida privatización, y una acelerada transnacionalización de las profesiones, que pone de manifiesto el impacto de la globalización en el sector.

Como resultado de estos cambios, el concepto mismo de "universidad" está también experimentando una transformación probablemente irreversible. La clásica trilogía funcional docencia, investigación y extensión está siendo cada vez más afectada en la producción de conocimiento y en el proceso de formación de los estudiantes, de manera que surgen cada vez más dudas acerca de su utilidad real.

\section{Primera parte: características del estudio}

Esta parte inicial comprende la descripción general del estudio que se realizó entre abril de 2005 y marzo de 2006, con el auspicio del Consejo Superior de Educación (CSE) de Chile. En esta parte se identifica el problema objeto del estudio: el examen de los "modelos" de gestión institucional que se están conformando en 
un grupo de universidades pertenecientes al Consejo de Rectores. Se pasa somera revista a la experiencia chilena antes de exponer los objetivos del estudio y su metodología.

\section{La problemática}

Los fenómenos descritos, combinados de variadas formas según las experiencias nacionales, están ejerciendo una aguda presión sobre la forma en que las instituciones de educación superior, especialmente las universidades, deben ser conducidas y gestionadas, puesto que muchas de las antiguas y más tradicionales prácticas y modelos de gestión universitaria no resultan adecuados para enfrentar los desafíos que plantea el cambiante contexto en que ellas están llamadas a cumplir su misión institucional y sus responsabilidades sociales.

Los sistemas y procesos de acreditación institucional, incluida la experiencia que se está desarrollando en Chile, constituyen una modalidad ampliamente utilizada para evaluar la eficiencia y la eficacia de las respuestas que las universidades otorgan a estos desafíos, incluyendo la evaluación de los controles de calidad de su gestión institucional en una perspectiva de autorregulación. Esta gestión ha pasado a ser de especial relevancia en la conducción de las universidades públicas, especialmente en aquellas cuyo carácter público proviene de su condición corporativa estatal. Esta preeminencia de la gestión se debe a diversos factores, entre los cuales se pueden destacar los siguientes:

\section{El desafío de la competitividad}

El sistema de educación superior, independientemente de si puede ser calificado de incipiente o desarrollado, tiene como uno de sus rasgos definitorios la incorporación significativa de instituciones privadas, cuyas carreras profesionales se ajustan, tanto en cupos como en perfiles de salida, a las presiones de demanda con un grado de oportunidad y flexibilidad reconocidas, que en gran medida se 
ven favorecidas por la capacidad de gestión empresarial con que se conducen estas instituciones. La gestión empresarial se aplica, en este sentido y de manera especial, a la utilización de los mecanismos e instrumentos financieros, a la aplicación de las políticas de reclutamiento y control del desempeño del personal académico, y a la gestión del marketing institucional. Todo lo anterior se resume en un sistema de toma de decisiones muy ágil en los procedimientos y muy centralizado en el más alto nivel corporativo de la institución, que suele corresponder a los niveles de su propiedad. Todas estas manifestaciones de efectividad conforman un persistente desafío que proviene de la creciente competitividad dentro del sistema, desafío que es marcadamente fuerte respecto de las universidades públicas que, en general, han aplicado, en forma muy limitada y con escasa convicción, fórmulas de gestión empresarial para su administración.

\section{La responsabilidad por los fondos públicos}

En el caso de las universidades públicas, las que reciben desde el Estado aportes directos de fondos públicos, se ha empezado a ejercer una creciente presión por aproximarse a sistemas de gestión más eficientes. Estando las instituciones del sistema de educación superior en condición de autonomía y con el reconocimiento político de la credencial con que estas universidades tienen "derecho" histórico a este aporte directo de financiamiento estatal, la responsabilidad por el uso de los fondos públicos no tiene fuerza legal. En consecuencia, se trata de una presión de hecho que proviene de los órganos jurisdiccionales del Estado y que se ejerce por medio de diversos conductos. Uno de ellos es el señalamiento del contraste entre el aumento sostenido de los fondos públicos canalizados a estas instituciones y el limitado mejoramiento de los indicadores de eficiencia que ellas pueden exhibir, con lo cual se está poniendo al descubierto, ante el público en general, que el rendimiento de estos fondos se vería asegurado si las instituciones mejoraran significativamente su gestión institucional. 


\section{Las exigencias de los fondos concursables}

El acceso a recursos públicos mediante los fondos concursables -que premian la capacidad de formular, presentar y ejecutar proyectos académicos dentro de un marco claro y ampliamente conocidopresiona hacia el mejoramiento de la gestión por el incentivo financiero. Es un hecho, por ejemplo, que la exigencia de que el proyecto se inscriba dentro de un diseño estratégico institucional se ha convertido en un eficaz mecanismo para que las instituciones formulen planes estratégicos de desarrollo, lo que repercute en una mejoría del sistema de gestión institucional. La exigencia de fondos de contrapartida no parece haber sido un incentivo igualmente poderoso, pues aquí las instituciones suelen optar por elevar el nivel de endeudamiento más que por controlar hacia adentro la eficiencia de la gestión.

\section{Las exigencias de los procesos de certificación}

Los procesos de certificación -que progresivamente se han institucionalizado en el sistema, ya sea por lo que respecta a la autonomía, en el caso de las nuevas universidades privadas, o de la acreditación institucional, en el caso de la universidades autónomashan sido un factor decisivo para el mejoramiento de la gestión de las instituciones.

En el primer caso, esta presión hacia la buena gestión se encauza a través del control sobre el avance y cumplimiento efectivo del proyecto institucional, de manera que es prácticamente imposible obtener la certificación de autonomía si la gestión institucional no se extiende eficazmente a los planos corporativo, estratégico, académico y financiero; en el segundo, la acreditación institucional obliga a las instituciones a demostrar que poseen una gestión que asegure el control de la calidad de sus procesos, incluyendo la capacidad de autorregulación, que conduce al reconocimiento de fallas que deben y pueden ser subsanadas por la propia institución. A esto se añade la exigencia de demostrar que hay igualmente una gestión adecuada de la docencia de pregrado. 


\section{La cultura modernizante}

Un último factor, no por ello es menos importante, es el progresivo afianzamiento de una cultura de modernización universitaria, proceso claramente notorio en el discurso predominante en las esferas de los académicos y de las autoridades universitarias. Los ámbitos donde la cultura modernizante ha hecho apreciables avances son variados y comprenden áreas como las siguientes: (i) modernización y reforma curricular; (ii) incorporación de las tecnologías de información y comunicaciones (TIC) en los procesos de enseñanza aprendizaje; (iii) agilización de la gestión institucional en facultades y departamentos para permitir presentaciones exitosas a las licitaciones de estudios y consultorías, y (iv) clima organizacional favorable a la instalación de procesos de autoevaluación.

En razón de lo expuesto, el CSE estimó pertinente incluir el examen de los modelos de gestión que se están institucionalizando en las universidades públicas dentro de su programa de estudios del sistema de educación superior en Chile, considerando la creciente importancia que tiene el diseño de un adecuado modelo de gestión para la conducción superior de las instituciones.

\section{La experiencia chilena}

El proyecto piloto, promovido por el gobierno con el apoyo del Banco Mundial y ejecutado por la Comisión Nacional de Acreditación (CNAP), ha llevado a cabo varios ciclos de acreditación institucional dirigido a instituciones autónomas. Este estudio se circunscribe a las instituciones que fueron acreditadas en el primer ciclo.

En la experiencia nacional las instituciones son evaluadas por medio de un proceso que combina evaluación interna y externa en dos áreas obligatorias y comunes: la gestión estratégica institucional y la de docencia, pudiendo hacerse extensiva la evaluación a una o más áreas específicas, según la voluntad de la institución que se somete a acreditación. La CNAP ha terminado 
procesos de acreditación con universidades autónomas, tanto de aquellas que reciben financiamiento directo del Estado, y que forman parte del Consejo de Rectores, como de aquellas que, sin recibirlo, se califican como instituciones "privadas".

Atendiendo que las decisiones de la CNAP, como agencia acreditadora, se expresan y se fundamentan, para cada institución, en un acuerdo público (que incluye consideraciones sobre la gestión institucional y de la docencia, dos áreas obligatorias, como se ha dicho previamente), existe en esos acuerdos un valioso acopio de información que incide en la forma como las instituciones están haciendo frente a los desafíos de conducción y gestión en el actual contexto de cambiosii ${ }^{\text {ii }}$.

\section{Objetivo del estudio}

En este estado de cosas, es posible abordar la tarea de organizar, sistematizar y analizar la información empírica proveniente de la actividad de la CNAP, con vistas a identificar y revisar críticamente los modelos de gestión puestos en práctica por las universidades y proponer algunas líneas de mejoramiento de la gestión institucional.

\section{Descripción del estudio}

\section{a. Ámbito y temas del estudio}

El estudio se refiere al análisis de los modelos de gestión institucional de las universidades del Consejo de Rectores. Al delimitar el trabajo a este grupo de universidades se obtiene un cierto marco institucional común que facilita el examen comparativo. Esta es una consideración

ii Una vez que se identificaron las universidades del primer ciclo de acreditación, se tomaron las pautas cualitativas de la CNAP y se hipotetizó que era probable encontrar problemas de gestión en un conjunto de áreas críticas o problemáticas, lo que arrojó diez tópicos donde era probable encontrar indicaciones acerca de dificultades o desafíos de gestión que no estaban siendo adecuadamente enfrentados por las instituciones, de acuerdo con las observaciones hechas por la CNAP para cada una en sus acuerdos de acreditación. 
que permite, además, abordar la tarea dentro de las limitaciones de tiempo y de recursos de que se dispone $e^{\mathrm{iii}}$.

Siguiendo, en general, la estructura de la pauta de criterios de la CNAP para la evaluación institucional tendiente a la acreditación, se pueden identificar tópicos (áreas de desempeño) para examinar la gestión institucional:

(i) La cautela o resguardo de la misión.

(ii) La estrategia de desarrollo.

(iii) La estructura organizacional.

(iv) La gestión económica.

(v) La gestión de la información.

(vi) La gestión de los recursos humanos.

(vii) El crecimiento cuantitativo.

(viii) La gestión de las carreras.

(ix) La gestión de las sedes.

(x) La gestión de la investigación y vinculación al medio.

Para los objetivos del estudio, estos temas se formulan en términos de problemas o exigencias de gestión. Esto quiere decir, por ejemplo, que el tópico "cautela de la misión" se formula como la necesidad de enfrentar este desafío desde la perspectiva de la gestión. En otras palabras, la pregunta que se hace a los datos institucionales es si el modelo presenta como problema la cautela de la misión. El supuesto es que todos los modelos se abocan a resolver los tópicos señalados. Lo que interesa es saber dónde el tema específico pasa a ser "problema", en el sentido de que allí se está en presencia de un desafío que el modelo de gestión no ha resuelto aún o no resuelve bien.

Las estructuras y procesos incluidos en los tópicos del estudio se analizan según los siguientes criterios (modalidades) transversales de gestión:

iii Una línea de investigación a partir de este estudio posibilitaría ampliar su cobertura a otros casos de instituciones. 
(i) Reglamentos - cultura organizacional.

(ii) Centralización - descentralización.

(iii) Autorregulación - control.

(iv) Participación - efectividad.

El principal aporte de estos criterios transversales es generar dos matrices que ayuden a identificar configuraciones características (cuadrantes) de gestión.

\section{b. Modalidad y secuencia del estudio}

El estudio analizó los procesos y estructuras de gestión en las universidades de Consejo de Rectores que han obtenido un pronunciamiento de acreditación por parte de la CNAP en el primer ciclo de acreditación realizado por esa entidad. Ese ciclo abarcó a un grupo amplio de instituciones de educación superior autónomas, incluyendo a dos institutos profesionales que no han sido incluidos en el estudio. Del conjunto de universidades que se inscribieron para ese primer ciclo, hay tres establecimientos privados que también han sido excluidos de este estudio. De las 13 universidades que son objeto del presente estudio, hay siete estatales y seis públicas no estatales. Entre estas últimas, cuatro universidades católicas y dos no católicas y regionales creadas por ley (Universidad Austral de Chile y la Universidad de Concepción).

Tabla 1: Universidades en la muestra del estudio

\begin{tabular}{lcc}
\hline Institución & Periodo de & Condición* \\
& Acreditación & \\
\hline
\end{tabular}

Universidad de Chile

Pontificia Universidad Católica de Chile

Universidad de Concepción,

Pontificia Universidad Católica de Valparaíso

Universidad Austral de Chile

Universidad de Magallanes

Universidad de Talca

Universidad de Tarapacá

Universidad de La Frontera

Universidad de Santiago de Chile

Universidad de Valparaíso

\section{(7)}

(7)

e

$\mathrm{p}$

$\mathrm{p}$

$\mathrm{p}$

$\mathrm{p}$

$\mathrm{e}$

e

$\mathrm{e}$

e

e

e 
*pública estatal (e); pública no estatal (p)

El estudio de los modelos de gestión de estas universidades descansa en dos fuentes principales de información documental:

- Los acuerdos de acreditación, que se examinan preponderantemente a la luz de los tópicos identificados en la sección precedente, reproducidos íntegramente en la página web de la CNAP. Tienen, por tanto, el carácter de información pública. El presente trabajo da cuenta del análisis efectuado con este material.

- Los informes de evaluación institucional preparados por las universidades para los fines del proceso de acreditación, que contienen una sección detallada sobre la gestión. Ellos permiten acceder a una información muy valiosa desde el punto de vista de la descripción y análisis que la propia institución realiza sobre su gestión. Para los efectos del estudio, se obtuvieron, a través del CSE, las debidas autorizaciones para la lectura de estos informes ${ }^{\mathrm{iv}}$.

\section{Segunda parte: Análisis de problemas de gestión en los acuerdos de acreditación}

En esta segunda parte del informe se incluye el análisis de los contenidos de los acuerdos que la CNAP adoptó al conceder la acreditación institucional al grupo de universidades del Consejo de Rectores que se presentaron voluntariamente al primer ciclo de acreditación. La revisión de cada acuerdo se hizo conforme a la

iv La codificación de sus contenidos en los capítulos relativos a la gestión estratégica institucional se realizó conforme a la matriz que se presenta en el Anexo. Originalmente, se había previsto la recolección de información adicional de modo directo entre los actores institucionales. A tal efecto se preparó, con la colaboración de Paula Mena, experta del CSE, un cuestionario que se envió a las instituciones por correo electrónico en diciembre de 2005. La gran mayoría de las instituciones acusó recibo de este documento. Sin embargo, a mediados de marzo de 2006 se había recibido una mínima cantidad de cuestionarios, por lo cual hubo que avanzar prescindiendo de esta información. 
pauta de áreas de problemas (tópicos) que la CNAP especificó para cada institución. De esta forma, se obtuvo la mirada externa especializada de la agencia acreditadora acerca de los procesos y mecanismos internos que aseguran la calidad de la gestión institucional. Asimismo, en esta parte se examina la muestra de instituciones incluidas en el estudio.

\section{Análisis de los acuerdos de acreditación: Las áreas problemáticas}

El análisis de los acuerdos de acreditación se hizo registrando las observaciones que la CNAP formuló, en cada uno de los casos, respecto de los puntos que mostraban algún tipo de alcance sobre gestión institucional. Al codificar sus observaciones, según los diez tópicos, se pudo construir una matriz de problemas de gestión cruzando los diez tópicos antes señalados con las instituciones.

\section{a) El análisis general de la muestra de instituciones}

La tabla 2 resume la totalidad del panorama registrado en la lectura de los acuerdos de acreditación para las instituciones de la muestra en estudio. El primer comentario es acerca de la variabilidad de los resultados, es decir, del número y distribución de las observaciones por institución. Esto apunta a una importante hipótesis, según la cual habría en las instituciones del Consejo de Rectores una variedad de modelos de gestión, según el patrón de lo que aparece como punto de debilidad en esta materia. 
CALIDAD EN LA EDUCACIÓN N² 24, julio 200677

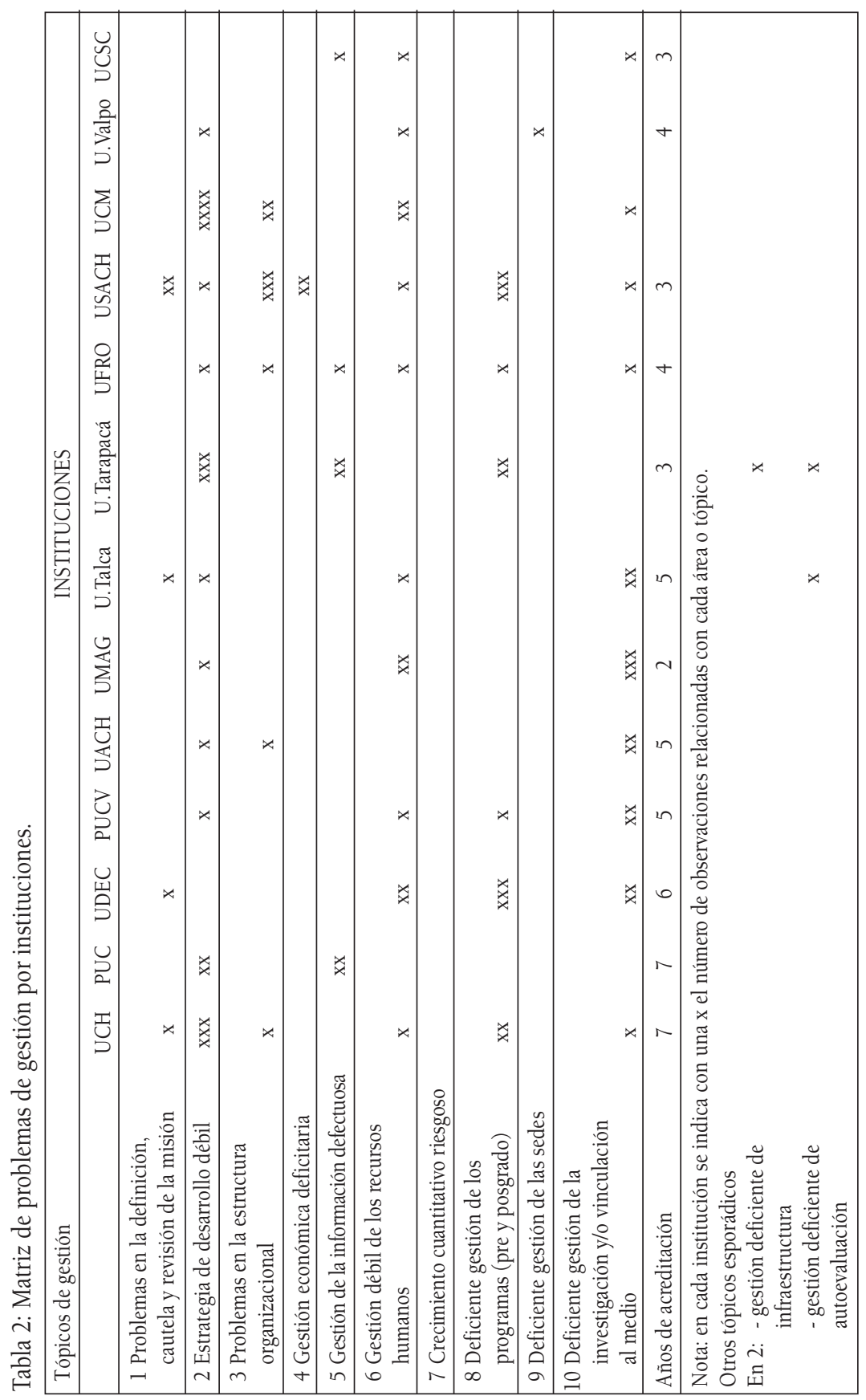


En el conjunto de instituciones incluidas hay una buena representación de universidades del Estado, tradicionales, derivadas, privadas antiguas, católicas, católicas pontificias, entre otras, de manera que se tiene una representatividad razonable. Se indican los periodos de acreditación solamente para mostrar que también aquí hay una diversidad muy grande y se incluye una columna que indica cuál es la condición institucional de estas universidades públicas, estatales y no estatales, que reciben aportes directos de fondos fiscales.

\section{b. El análisis por tópicos de gestión}

La información pertinente se presenta en la tabla 3. Se han contabilizado las menciones registradas en los informes de acreditación según las áreas problemáticas para la gestión. Ordenar los datos de esa forma permite jerarquizar problemas según su orden de importancia.

Hay algunas grandes áreas problemáticas en la gestión institucional, a juzgar por la distribución de las menciones que se encuentran en los informes de acreditación. Estas áreas son, en este mismo orden:

(i) Debilidad de la estrategia de desarrollo institucional, lo cual puede estar apuntando a dos tipos de problemas: uno sería la fragilidad de la difusión de la estrategia en el conjunto de la comunidad universitaria; el otro, una deficiente formulación de la estrategia en términos de su falta de concreción en metas, plazos indicadores de resultados y responsables institucionales.

(ii) Deficiente gestión de la investigación y de la vinculación con el medio, aspectos que en el esquema de acreditación de la CNAP están muy relacionados. Hay un grupo relativamente reducido de instituciones donde la investigación tiene un peso con cierta densidad en la estructura institucional, lo cual estaría indicando que la gestión en el grueso de la muestra de instituciones es débil, porque la actividad de investigación es así.

(iii) Defectuosa gestión de la docencia, en todos sus niveles. La aparición de este tópico en el tercer orden de importancia 
debiera llamar a la reflexión, porque ello indicaría modelos ineficientes en un aspecto sustancial de la universidad, como es la docencia de pre y posgrado.

(iv) Problema de gestión respecto de los recursos humanos, en directa relación con el reclutamiento, evaluación, jerarquización, perfeccionamiento, renovación y desvinculación en las plantas académicas de las universidades.

(v) Problemas asociados con el diseño de la estructura organizacional adecuada para la gestión institucional.

Tabla 3: Problemas de gestión según orden de importancia

\begin{tabular}{lcc}
\hline Tópicos de gestión según problemas observados & $\begin{array}{c}\text { Número de } \\
\text { menciones }\end{array}$ & Ranking \\
\hline Problemas en la definición, cautela y revisión de la misión & 4 & 7 \\
Estrategia de desarrollo débil & 21 & 1 \\
Problemas en la estructura organizacional & 10 & 5 \\
Gestión económica deficitaria & 3 & 8 \\
Gestión de la información defectuosa & 6 & 6 \\
Gestión de los recursos humanos & 14 & 3 \\
Crecimiento cuantitativo riesgoso & 0 & 0 \\
Deficiente gestión de los programas (pre y posgrado) & 13 & 4 \\
Deficiente gestión de las sedes & 2 & 9 \\
Deficiente gestión de la investigación y/o vinculación al medio & 16 & 2 \\
\hline
\end{tabular}

Fuente: Elaboración propia a partir de los acuerdos de acreditación CNAP.

Respecto de las áreas problemáticas que están entre las cinco primeras del ranking, es posible adelantar una interpretación relativamente razonable, en el sentido de que todas ellas apuntan a procesos de maduración lenta. En efecto, formular una estrategia de desarrollo adecuada, efectuar una eficiente gestión de la investigación o de los recursos humanos, así como de los programas, o la creación de una estructura organizacional adecuada, son procesos lentos que, en cuanto tales, tienen tiempos diferidos y plazos largos para empezar a lograr resultados. Ellos implican dinámicas de intervención y gestión con espacios temporales muy amplios para la retroalimentación. Eso hace que, a medida que el proceso se desarrolla, haya una ocurrencia de cambios en insumos, en operaciones o en agentes. En efecto, es frecuente que agentes 
que tomaron decisiones con gran impacto en la intervención específica de que se trate ya no estén cuando empiezan a producirse los resultados de sus decisiones.

Por otra parte, existe la probabilidad de que, en algún punto de estos procesos largos, se empiecen a producir efectos no deseados ni anticipados, algunas veces virtuosos y otras veces viciosos. Ejemplos característicos de estos procesos son la formación de una masa crítica de académicos, el desarrollo de la investigación de cierto nivel, la puesta en régimen de una carrera, el desarrollo de competencias reconocidas por la comunidad académica en áreas determinadas, que es algo así como la construcción del prestigio profesional, entre otros.

Es notoria la necesidad de hacer una reflexión amplia acerca de cómo gestionar estos procesos cuya lentitud está conformada desde el núcleo de la cultura académica. El problema supone una gran paciencia institucional y obliga a pensar en la estabilidad de los elencos directivos, exige un diseño de indicadores adecuados a la lentitud de la maduración de estos procesos y requiere de una estrategia para el manejo inteligente de los efectos no anticipados, propios de la lenta maduración.

\section{c. Análisis por áreas problemáticas en la gestión}

Es posible examinar ahora, someramente, cada una de estas áreas, siguiendo el ranking de las frecuencias de las menciones respectivas observadas en los acuerdos de acreditación. El dato empírico de la gravitación de cada área ha dado pie para avanzar en algunas interpretaciones, que permiten hacer preguntas acerca de las prácticas de la gestión institucional en las universidades estudiadas.

\section{(i) Formulación de la estrategia de desarrollo}

Esta es el área problemática más gravitante. Hay aquí problemas que tienen que ver con la especificación de fines como eje del desarrollo del proyecto institucional y, en el caso de que estos fines 
sean expresados, cómo estos se traducen en metas, porque la meta es un fin alcanzable dentro de un tiempo estimable o medible.

La estrategia de desarrollo obliga a tener en el gran diseño del proyecto institucional alguna indicación de cómo una universidad o institución va a abordar el problema de la gestión de los procesos académicos de lenta maduración. Todo indica que las instituciones han avanzado en la identificación de orientaciones estratégicas generales, pero no es igualmente claro que éstas hayan desembocado en planes que puedan guiar en forma operativa los procesos de gestión estratégica. En muchos casos, las orientaciones son más bien indicativas de una suerte de trayectoria genérica que debe ser traducida en una agenda de gestión trazada según planes, que posibiliten una formulación más concreta y más dura de lo que se persigue en términos de estrategia de desarrollo de las instituciones.

(ii) Problemas en la gestión de la investigación Subrayan el carácter de la investigación como un proceso lento y permiten destacar algunos puntos relevantes para la discusión de la gestión de la investigación. El primero de ellos es el tema del aprovechamiento de las oportunidades desencadenantes de la investigación. En efecto, el desarrollo de procesos o masas críticas de investigación es muy dependiente del aprovechamiento de ciertas oportunidades que se presentan, ya sea porque se abren contactos con determinados investigadores, porque se establecen nexos en el exterior o se identifican problemas que tienen una fuerte gravitación coyuntural en el país.

Un segundo punto se refiere al efecto "interno" de la vinculación con el medio, que se traduce en la necesidad de manejar adecuadamente la tensión entre la disciplina y la consultoría. De una forma u otra, la gestión de la investigación exige tener claridad acerca de cuántos de aquellos contactos con el medio son alimentadores del desarrollo de la disciplina y cuántos son demandas de competencias para la asistencia técnica. En general, 
esta es una tensión no resuelta. Otro punto es la necesidad de filtrar, de manera rigurosa, el papel de las tesis como proyectos de investigaciones reales, que tengan impacto efectivo dentro de las universidades. Las tesis son necesariamente protoproyectos de investigación de largo aliento; hay que partir de la base de que, excepcionalmente, se encuentran trabajos de tesis como aportaciones significativas desde el punto de vista del impulso a la investigación.

Por último, existe el problema de vincular investigación y docencia de manera efectiva. Desde el punto de vista de la gestión, es indispensable tener cierta claridad acerca de cuál es la docencia (tanto de pre como de posgrado) que exige un nexo con la investigación que es necesario fortalecer o establecer, según el caso. No toda la investigación que se hace en la institución sirve para la docencia y alguna sirve sólo para cierto tipo. Hay demandas pedagógicas y también demandas investigativas de docencia que, estrictamente hablando, no exigen para su cumplimiento el ajuste a patrones de proyectos de investigación disciplinaria en términos rigurosos, sino que apuntan a otro tipo de investigación como es, por ejemplo, el análisis institucional.

(iii) Debilidad en la gestión de los recursos humanos Hay aquí involucrado un proceso de lenta maduración como es la formación de la masa crítica académica, que exige eficiencia en la gestión de áreas como la política de reclutamiento de los académicos, los mecanismos de reciclaje para hacer posible la actualización docente y la política de desvinculación que posibilita la renovación de las plantas académicas. Este es un sector generalmente mal administrado por las universidades.

Otro tema relacionado es el tratamiento del peso del prestigio en la progresión y reconocimiento de los académicos. Las universidades son instituciones que, en su cultura interna, valoran especialmente este rasgo cualitativo que se expresa en reconocimiento tanto de los pares de la comunidad interna como 
de las comunidades disciplinarias externas. Esto conduce a que la introducción de incentivos en la gestión de los recursos humanos tenga una cierta deriva de rendimiento decreciente, porque hay un punto en que, independientemente de cuál sea la frecuencia, los montos, las modalidades y los incentivos, el peso del prestigio hace que éstos se tornen irrelevantes. Este es un problema de compleja solución, pues no es fácil desarrollar una política de recursos humanos que dé cabida a un aspecto tan eminentemente cualitativo como es el peso del prestigio, que, por cierto, repercute en la legitimación de la evaluación del rendimiento y la productividad de los académicos.

También hay que mencionar, dentro de esta área crítica, la tensión no resuelta entre una evaluación que descansa en asuntos de forma v/s una que descansa en sustancia. Pareciera que hay una fuerte tendencia a instalar procesos que son sólo evaluaciones formales, que dependen, a veces excesivamente, de los formularios, de la gradiente jerárquica de las instancias que participan o de las consultas que se hacen a los alumnos, sin darle mucha cabida al aspecto más sustantivo de la calidad académica del desempeño, que es la parte más complicada de la evaluación del rendimiento.

\section{(iv) Debilidades en la gestión de los programas}

La gestión de las carreras entra de lleno en el tema de la docencia, pero lo que interesa en este estudio no es la calidad de ésta, sino la forma en que aquéllas son administradas. Como ocurre en otras de estas áreas problemáticas, también hay aquí un proceso de maduración lenta, por el hecho de que el alumno es un agente de su propia formación. Mediante un proceso de selección más o menos estricto, una persona ingresa a la universidad y empieza un proceso que lo va acompañar por varios años de su vida. Éste es, de suyo, lento y se expresa en el currículo que es el canon de la formación.

Entonces, la gestión de las carreras tiene que ser enfocada desde el punto de vista de la actualización y la innovación curricular necesarias para hacerse cargo del proceso formativo. No hay 
prácticamente universidad en la cual no esté instalado el tema de la innovación curricular; pero, en muchos casos, lo que hay es más un discurso de innovación curricular sin que haya el nexo fundamental entre lo declarado y el desarrollo del proceso lento, que es la formación del alumno.

Otro problema que suele aparecer vinculado a la gestión de las carreras es la escasa validez de las prácticas para desescolarizar el aprendizaje, que significa abrir, en el proceso de formación y en la innovación curricular correspondiente, espacios para que el alumno ejerza su propia autonomía intelectual. Las instituciones suelen más bien reproducir prácticas o modalidades de gestión de las carreras, donde se busca generar el mejor de los entornos para que el alumno casi no tenga que tomar decisiones respecto de, por ejemplo, un problema intelectual que le plantean una asignatura, una evaluación, un determinado proyecto, un ensayo o una prueba de laboratorio. En la universidad el estudiante tiene que cultivar su propia autonomía y, para ello, es imprescindible desescolarizar el aprendizaje, enfatizar que la gestión de la carrera produce un cambio cualitativo desde el entorno escolar al entorno universitario.

Con esta exigencia cobra creciente importancia la acreditación. Ésta debe ser considerada como un capital para la inversión futura de la carrera, más que como una certificación de logros pasados. La gestión eficiente de las carreras tiene que proceder de esta manera.

(v) Dificultades para conformar la estructura organizacional Los dilemas envueltos en esta área tienen que ver, en general, con la participación segmentada de los estamentos. Todo parece indicar que la participación brota, se genera y se sostiene en áreas específicas, según sea la composición de los estamentos involucrados. No hay, al parecer, procesos participativos transversales que involucren a todos por igual, no hay una suerte de ciudadanía participativa en la universidad. Hay participación, pero en áreas bastantes especificas, donde los académicos, los 
funcionarios, los administrativos o los directivos, incluso los estudiantes, se involucran, pero no lo hacen respecto de la totalidad de la organización.

Es muy probable que esta participación segmentada vaya a persistir en el futuro y, en ese predicamento, el problema de gestión en la estructura organizacional es cómo manejar la autonomía hacia adentro, es decir, cómo controlar los espacios que, finalmente, quedan entregados al libre arbitrio de un docente que, por su capacidad o su experiencia, resuelve hacer las cosas de cierta manera, sobre todo en el desempeño de su docencia. Es claro que la libertad de cátedra incide en una dimensión que está dentro de las más clásicas definiciones del ethos de la universidad, en tanto institución, que tiene una irrenunciable vocación y compromiso con la libertad académica, tanto respecto de lo que se enseña como de lo que se investiga. Se trata, pues, de una autonomía interna que garantiza, en el nivel de cada académico, el derecho a la "búsqueda desinteresada de la verdad" y, en el nivel de la institución, el pluralismo intelectual. El tema central es el de la evaluación del desempeño académico, que es donde se resuelve finalmente la ecuación entre autonomía y responsabilidad, o, si se quiere, entre libertad y control. Como se verá más adelante, este aspecto de la evaluación del desempeño de los académicos es una de las materias más débilmente desarrolladas en la gestión institucional y compromete, a veces con seriedad, la viabilidad de las políticas de desarrollo de los recursos humanos de las universidades.

Cuántos espacios de autonomía interna de las personas permite la estructura organizacional tiene que ver no sólo con las prácticas y procedimientos de evaluación del desempeño, sino, también, con las prácticas de desescolarización del estudiante, para que éste sea efectivamente tratado como un agente autónomo. Esto apunta a la interdependencia entre la autonomía del profesor que enseña en su cátedra y la autonomía de sus estudiantes, cuyo aprendizaje debería ser un proceso de creciente ampliación de sus propios espacios de aprendizaje. 
En los campos de la gestión institucional suele darse mucha importancia a la autonomía como un rasgo institucional hacia afuera; pero, tanto o más importante, es que la universidad se dote de una estructura organizacional que permita la creación de espacios de autonomía hacia adentro. Esto no debe interpretarse en el sentido de minimizar la importancia de la autonomía externa de las instituciones; vale decir, la capacidad de adoptar decisiones estratégicas sin interferencias o presiones de agentes políticos externos, sean o no de carácter gubernamental.

La autonomía universitaria, entendida en este sentido externo, constituye un rasgo decisivo de la cultura académica más propia de las tradiciones europeas y, especialmente, latinoamericanas. Como tal, ha sido un factor importante en la creación de condiciones que permitieron el desarrollo "estratégico" de las instituciones, por cuanto la autonomía externa sustrajo a las universidades de las veleidades ideológicas de gobiernos muchas veces inestables y, por otra parte, en el caso de las universidades estatales, permitió dotarlas de recursos cuyos flujos y montos pudieron tener una cierta estabilidad no sujeta a las negociaciones de corto plazo con las autoridades gubernamentales.

No obstante, desde el punto de vista de la gestión organizacional, entendida en el marco de las prácticas modernas, es pertinente indagar sobre la forma en que se resuelve la ecuación fundamental entre autonomía y responsabilidad, especialmente en el caso de las universidades que se analizan en este estudio, que tradicionalmente han recibido aportes de fondos públicos, con razonable transparencia, pero sin controles efectivos acerca de su uso. Nuevamente, este tema se liga con la introducción de instrumentos de evaluación del desempeño institucional que permitan ponderar el grado de responsabilidad en el uso de los recursos recibidos. Si bien es cierto que, en la actualidad, es una materia de muy incipiente desarrollo en nuestro medio, es probablemente una de las áreas donde debiera producirse un cambio de la mayor envergadura en las prácticas de gestión estratégica de 
las instituciones y, como tal, debiera ser un campo de altísima prioridad para las políticas públicas en la educación superior del país.

Otro tema que surge en esta área es cómo combinar estructuras jerárquicas con estructuras planas. En las universidades hay áreas y tipos de procesos que son muy proclives a estas últimas, sobre todo aquellos donde prima el criterio de juicio de los pares. Como es sabido, este es un fuerte factor organizacional en las universidades; sin embargo, junto con ello, existen también las estructuras jerárquicas con las cuales suele generarse una tensión no resuelta en torno a la creación de liderazgo académico.

Es difícil pensar que una universidad esté avanzando hacia una estructura organizacional adecuada si no hay una creación de verdadero liderazgo académico, que es siempre una mezcla de posición jerárquica y trato igualitario entre pares. En esto no parece haber recetas: se trata de un problema que la institución tiene que resolver desde adentro. La construcción de liderazgo es también de lenta maduración. No necesariamente se resuelven los problemas cuando un comité de búsqueda trae un rector que tiene una gran reputación, pues eso no significa per se liderazgo académico en la organización a la cual se llega.

(vi) Debilidades en la gestión de la información

Hay áreas que son claramente menos problemáticas en las universidades estudiadas, pero que, no por ello, deben ser dejadas de lado en el análisis de la gestión institucional. Entre ellas aparece, en primer lugar, la gestión de la información, lo cual no deja de llamar la atención, considerando que ha habido un enorme esfuerzo por desarrollar las capacidades sistémicas en las universidades. Ciertamente, se ha hecho un gran progreso en la instalación de sistemas, la aplicación de programas computacionales actualizados, potentes y cada vez más amigables, y en la utilización de las técnicas de recolección. 
Con todo, hay que reconocer que, en distintos niveles, áreas, sectores y procesos de gestión de la universidad la información tiene un coeficiente de importancia distinto, pero no parece haber mucha finura para hacer esta distinción, que incide decisivamente en la forma cómo se producen los datos. Muchas veces la información parece producirse más como respuesta a estímulos externos que por constataciones genuinas de la necesidad de producirla desde dentro. Si se hace un balance de las razones para la instalación de las innovaciones, la mayoría de ellas ha sido por solicitudes externas; hay aquí todavía un tema pendiente que es el de la producción de la información donde realmente corresponda. Relacionado con lo anterior, existe un problema en cómo se selecciona y cómo circula la información, cómo y quiénes la usan, y todo ello se resume en quién retroalimenta a quién con los sistemas de información.

(vii) Problemas en la cautela de la misión El resguardo de la misión aparece como un problema de menor gravitación en este grupo de universidades. Aventurando una posible hipótesis, habría que señalar que, si bien la introducción de la reflexión en torno a la misión es relativamente nueva en las universidades latinoamericanas, las universidades públicas tienen una cierta sintonía con preguntas sobre su rol en la sociedad y su nexo político con ella. Son universidades que tienen un diálogo con el Estado a través de los recursos que reciben; entonces, es posible hipotetizar que en ellas se encarna una cultura institucional más sensibilizada a hacer preguntas sobre el compromiso institucional con el país.

Debemos atender a la importancia real de la misión en la institución, cual es su vigencia, su grado de socialización y efectividad. Una misión puede estar socializada en la comunidad universitaria, pero el grado en que ella es efectiva, más allá de una cierta comunidad de lenguaje y discurso, es asunto que depende fundamentalmente del gobierno corporativo de la institución. 
Es indispensable dar una mirada a la formación de las instancias corporativas. Tanto o más importante que la adopción de políticas generales o las decisiones sobre apertura o cierre de carreras, el resguardo de la misión tiene que ver con el alma de la universidad y debe ser asumido en la conformación del gobierno corporativo.

(viii) Problemas en la gestión económica

Aquí los problemas no se reducen sólo al manejo de los flujos financieros, sino que apuntan a las maneras de gestionar recursos públicos. Las universidades públicas del Consejo de Rectores son instituciones comprometidas, en principio, con una formación destinada al servicio y que asumen su responsabilidad social en la formación de profesionales. El asunto consiste en realizar la gestión económica de la institución en espacios cada vez más condicionados por las reglas del mercado, como es el caso de las profesiones.

Otro problema se refiere al manejo de la desigual distribución interna de recursos, esto resulta particularmente notorio en algunas universidades que, como producto de las políticas de autofinanciamiento, han usado una estrategia muy eficaz para generar recursos externos mediante asistencias técnicas o consultorías, o por la venta de proyectos en ciertas áreas "rentables" que conviven con otras áreas que son extremadamente deficitarias. Este es un tema no resuelto y plantea problemas en cómo se redistribuye y se maneja el desigual reparto interno de los recursos, cómo se ha de ponderar la productividad en la venta de servicios que tienen algunos equipos académicos v/s la incapacidad de otros por razones muchas veces no directamente bajo su responsabilidad.

Desde la perspectiva institucional, parece haber una tensión permanente entre la tendencia a la apropiación empresarial de la universidad v/s su apropiación académicav ${ }^{\mathrm{v}}$, como criterio para inspirar la toma de las decisiones más cruciales en la institución. El asunto es cómo se logra la combinación de esas dos lógicas y, al

v Se utiliza aquí la palabra apropiación en términos de "empoderamiento" (empowerment). 
respecto, es frecuente que las universidades oscilen entre una y otra, sin articular bien estas dos formas de empowerment.

\section{(ix) La gestión de las sedes}

Los problemas de crecimiento cuantitativo global -otro de los tópicos de gestión seleccionados para el estudio- no se observan en los acuerdos de la CNAP, debido, probablemente, a que las universidades analizadas están relativamente estabilizadas. En cuanto al tema de la gestión de las sedes hay al parecer dos dilemas y un desafío. El primero, en instituciones que tienen estrategias de crecimiento tentacular, es el de cómo se aborda el problema de descentración de la universidad, la cual pierde su centro de gravitación corriendo el riesgo de debilitar su sello institucional. Hay algunas de estas universidades que caen dentro de esta estrategia, pero es una situación poco frecuente.

El otro problema es el de cómo abordar y controlar eficientemente la seducción localista, expresada a través de los llamados de las "fuerzas vivas" de la región, que atraen a la institución ofreciendo instalaciones e infraestructura (normalmente un comodato por algún numero de años). Muchas veces, las universidades no responden en forma muy selectiva a esta seducción localista y aquí el tema critico de la gestión es cómo asegurar el carácter unitario de la calidad. Esto implica que cualquiera sea el estándar que se aplique en la localidad, la gestión institucional asegurará que la calidad de todo aquello que la universidad provea en sus ofertas académicas se ajuste al mismo estándar de calidad. Este es un problema extremadamente complicado de resolver y obliga a explicitar una política de gestión de las sedes que, por lo general, no es clara.

\section{Tercera parte: Modelos y problemas de gestión en los informes de autoevaluación de las instituciones}

En esta parte el análisis se enfocó en los informes de autoevaluación que cada institución debió preparar, conforme a pautas indicativas 
de la CNAP como parte fundamental del procedimiento establecido por la agencia acreditadora. A diferencia del análisis presentado en la parte precedente, aquí el acento está en la mirada interna que las instituciones hicieron sobre sus propios procesos y estructuras de gestión institucional. La dimensión que comparativamente relaciona las dos miradas es la caracterización de las áreas de problemas específicos de gestión con algunas variaciones menores que se explican al final de esta parte.

No obstante, un elemento de gran importancia para el objetivo del estudio, y que sólo podía obtenerse del examen de los informes de las instituciones, fue el análisis de temas "transversales" que, supuestamente, debían cruzar todas las áreas de problemas específicos de gestión. El análisis hecho desde esta perspectiva permitió configurar una trilogía central para perfilar "modelos" de gestión. Esta se obtuvo al priorizar tres áreas de problemas específicos por la amplitud de su rango y de su impacto en la gestión de las instituciones: la misión institucional, la planificación estratégica y la estructura organizacional de la gestión institucional. Este análisis transversal se presenta en los tres primeros apartados de esta parte.

El resto de la tercera parte está dedicado a examinar los puntos de vista de las instituciones respecto de las demás áreas de problemas específicos y a presentar, en el apartado final, algunas conclusiones y proposiciones que se derivan el estudio.

\section{Análisis de los temas transversales para la gestión institucional. Una trilogía central para los modelos de gestión}

En los documentos que las instituciones prepararon para su proceso de acreditación institucional hay algunos grandes temas centrales en las descripciones y evaluaciones de la gestión estratégica institucional, con los cuales puede iniciarse el análisis de los temas 
transversales, de acuerdo a la matriz que orienta a este estudio ${ }^{\text {vi }}$. Ellos configuran una suerte de trilogía fundamental para los modelos de gestión: el primero tiene que ver con la cautela o resguardo de la misión institucional; el segundo con el proceso de planificación estratégica y su producto, que es el plan de desarrollo institucional (PDI); el tercero se refiere a la estructura orgánica que la universidad ha establecido para su gestión institucional.

\section{(i) La misión institucional}

La cautela de la misión es responsabilidad de los cuerpos colegiados de alto nivel, especialmente de las juntas directivas, en las cuales existe representación externa que, en el caso de las universidades estatales, se materializa en los representantes del Presidente de la República ${ }^{\mathrm{vii}}$. En las universidades católicas esta integración de miembros externos procede por decisión del Gran Canciller que, a veces, tiene la facultad de designar a personas de su confianza como miembros externos.

En las universidades públicas que no son estatales ${ }^{\text {viii }}$ ni confesionales, la participación externa está reglamentada en los estatutos que conforman el cuerpo legal fundacional de la institución y se expresa en las personas de los socios fundantes de la universidad.

vi Es importante señalar que, dado el carácter general y mandatario de las áreas de gestión institucional y gestión de la docencia que obligadamente deben ser cubiertos en los procesos de acreditación institucional de la CNAP, hay algunos temas que, en la revisión de los informes preparados por las universidades estudiadas, estuvieron presente solamente en algunas de las instituciones. Por esta razón, los temas relacionados con la gestión de la investigación y la vinculación con el medio no se han revisado en esta sección.

vii La única universidad pública, no estatal, que tiene un representante del Presidente de la República en su órgano colegiado de más alto rango es la Universidad Técnica Federico Santa María (UTFSM). A diferencia de las estatales, donde estos miembros representan al Estado en cuanto "dueño" de la institución, en la UTFSM la reforma de estatutos que abrió la entrada a un representante del Presidente de la República asumió que este miembro externo "político" estaba destinado a actuar como facilitador del diálogo de la institución con la autoridad estatal.

viii Estas son, además de la UTFSM ya mencionada, la Universidad Austral y la Universidad de Concepción. Es preciso hacer notar que la UTFSM, a diferencia de las otras dos, no estuvo entre las universidades que hicieron su proceso de acreditación en el primer ciclo de la CNAP. 
Estas fórmulas de representación externa persiguen, formalmente, asegurar espacios para que, de una u otra forma, se manifiesten las opiniones y posturas de los propietarios en asuntos que inciden en aspectos fundamentales de la vida de la institución, como son las decisiones que se refieren al aseguramiento de la viabilidad económica y al de la fidelidad de la universidad a un núcleo de valores que plasman la ética, tanto secular como religiosa, que conforma la manera como la universidad se relaciona con su entorno social.

El supuesto normativo aquí descansa en que es el dueño de la universidad quien mayores responsabilidades tiene en lograr este doble aseguramiento de la viabilidad y de la fidelidad de la institución. El dueño (actor externo a la vida académica) es, entonces, el guardián preferente de la misión institucional.

El Estado aparece como un dueño poco celoso de su función cautelar. Controla de algún modo, pero no necesariamente con todo el rigor del propietario, la viabilidad económica de sus universidades mediante la supervisión del endeudamiento financiero y de concurrir, con poder de veto a través de sus representantes, a la adopción de decisiones que pueden afectar al patrimonio de la universidad. Esto se refleja en que la función de sus representantes se ejerce casi exclusivamente en las decisiones económicas, donde el voto favorable de estas personas es requisito indispensable para la validez del acuerdo de que se trate.

\section{(ii) La planificación estratégica}

a) Los planes de desarrollo

Todas las instituciones tienen, en diversos grados de especificación y de proyección temporal, planes de desarrollo destinados a orientar el devenir de la universidad articulando una doble dinámica: (1) la que proviene de la misión institucional y de la correspondiente visión que la universidad ha diseñado de sí misma en un horizonte de mediano o largo plazo, y (2) la que proviene de los desafíos y oportunidades del entorno. 
Normalmente se encuentra que, en la base de la formulación del plan, hay algún ejercicio de planificación estratégica que suele adoptar el formato FODA (Fortalezas, Oportunidades, Debilidades y Amenazas). Los planes han surgido a veces de las exigencias externas planteadas por agencias que han sido capaces de exigir diseños de planificación institucional como requisitos para la adjudicación de beneficios o recursos públicos administrados desde el sector gubernamental.

En general, las universidades que se denominan "derivadas", y que se iniciaron autónomamente a principios de la década de los 80, han sido las principales protagonistas de esta planificación estratégica inducida desde el exterior. Las universidades llamadas "tradicionales", en cambio, pasaron por escenarios donde el planeamiento estratégico de la institución se instaló como una preocupación y una tarea que las respectivas comunidades académicas debieron enfrentar antes de la intervención por parte del régimen militar. Estos escenarios correspondieron a las fases históricas de los proceso de reforma de las universidades a fines de los 60 y comienzos de los 70. Pero, aún en estos casos, puede decirse que fue a partir de los 80 cuando esta actividad tiende a hacerse más sistemática y tecnificada.

b) Desajustes entre objetivos y aseguramiento de las metas Puede observarse que, en general, existe aún una brecha no cubierta entre la formulación de los grandes trazados estratégicos, que sirven para orientar el rumbo general de la institución, y la especificación de metas cuantificables e indicadores que permitan el seguimiento continuo del grado de logro de esas metas. También suele ocurrir que, cuando se avanza en la especificación de tales metas, se hace más bien un ejercicio formal que un desarrollo sistémico del proceso que conduce a la meta. Un ejemplo puede ilustrar esta circunstancia.

Prácticamente todas las instituciones tienen entre sus orientaciones estratégicas (y algunas entre sus metas cuantificables) el mejoramiento de la formación de sus académicos. Para ello se 
prevé algún tipo de acción (eventualmente un programa) con el fin de incentivar la contratación de posgraduados (especialmente doctores) o de facilitar la obtención de grados superiores a los académicos de la universidad. En algunos casos se dispone incluso de programas de financiamiento de becas de posgrado que alcanzan montos significativos de recursos para inducir a la formación doctoral a los académicos jóvenes. Lo que normalmente no se encuentra es el conjunto de medidas y acciones tendientes a asegurar la reinserción efectiva de los académicos que exitosamente cumplieron con su programa de posgrado.

En la práctica, la estrategia de reinserción pasa a ser un asunto entregado en buena medida a las acciones individuales. El resultado es la frustración y la pérdida de compromiso que son, indudablemente, las condiciones para la emigración laboral. Este es un caso de priorización de un objetivo estratégico (mejorar la dotación de académicos altamente calificados) que falla en la mantención de la meta alcanzada por la institución por debilidad de las acciones de seguimiento sistémico de ese logro.

Algo similar se observa en el mejoramiento de las condiciones y recursos tecnológicos para modernizar las prácticas docentes. El objetivo estratégico de la inversión en este tipo de equipos y servicios, que frecuentemente se alcanza en el plano de la implementación física, termina siendo acompañado por una baja y a veces muy baja tasa de utilización efectiva de estos recursos tecnológicos.

Si el trabajo macroinstitucional de formulación del Plan de Desarrollo Institucional (PDI) no va acompañado de las actividades de "primer piso", que suponen acciones en las unidades académicas (para asegurar una efectiva reinserción laboral o una aceptable tasa de utilización de equipos y servicios disponibles, según los casos reseñados), el ajuste y la coherencia entre los objetivos y el aseguramiento de las metas se ven gravemente comprometidos. Lo que se perjudica es la gestión sistémica de la institución. 
c) Los agentes responsables de la planificación.

Otro aspecto que se observa con cierta nitidez es la enorme variabilidad de concreción orgánica de la función de planificación estratégica. Las así llamadas direcciones de planificación, o sus equivalentes funcionales, se articulan en muy diversas estructuras y procesos de gestión de la función. Por lo general, suelen encontrarse estas unidades en el seno de los equipos rectoriales, con lo cual terminan siendo más bien oficinas de asesoría al rector que entidades efectivamente depositarias de la inteligencia institucional.

En los procesos de designación de las autoridades, especialmente si hay de por medio procesos electorales, estas unidades de planeamiento de la institución son discretamente redireccionadas para resaltar los logros de una determinada gestión rectorial. El punto de fondo aquí es la necesidad de institucionalizar esta función y, si bien ello admite variantes de acuerdo al tejido organizacional de cada universidad, el objetivo que debiera ser plenamente compartido es el logro de instalación de una unidad donde se produzca y se cautele la sistematicidad del diseño estratégico de la institución, con las debidas instancias de accountability del equipo que asuma la conducción y la administración de esta unidad de inteligencia.

Con esto se aseguran los resguardos para evitar que estas direcciones de planificación se conviertan en incubadoras de tecnocracias de gestión académica, que siempre resultan dañinas para el sano desarrollo de la universidad.

\section{(iii) La estructura orgánica para la gestión}

En relación con el segundo tema transversal, que se vincula con las estructuras orgánicas de gestión institucional, el análisis puede abordarse desde una doble perspectiva. La primera está centrada en los procesos de regulación normativa de la institución; la segunda, en la estructura de gestión. 


\section{a) Los procesos de regulación}

La perspectiva de la regulación se construye en un doble aspecto: el primero pone el acento en la fuente de las normas, atendiendo a si ellas se originan en el entorno de las instituciones, en cuyo caso se trata de normas originadas y cauteladas por agentes externos a las universidades; o si ellas se originan en el seno de la institución, en cuyo caso se trata de normas que provienen de procesos de autorregulación.

El segundo aspecto tiene que ver con la naturaleza de la regulación, atendiendo a si ella está formalizada en cuerpos explícitos (regulación formal) o si, más bien, se inserta en la cultura universitaria dando origen a códigos de conducta que tienen una efectiva vigencia, pero que no están codificados en un cuerpo reglamentario; pertenecen a la esfera de las prácticas y patrones aceptados de comportamiento, culturalmente aprendidos, como parte del ethos universitario.

\section{b) Las estructuras de gestión}

La perspectiva de la estructura de gestión toma en cuenta también un doble aspecto. Por una parte, recoge la dimensión de centralización o descentralización de ella y, por otra, los procesos de participación de uno o varios estamentos académicos.

c) Los campos de análisis: procesos de regulación y estructuras de gestión

Habiendo precisado las dimensiones implicadas en estas dos perspectivas, se pueden construir los campos que se originan al combinar tales dimensiones para cada una de las perspectivas señaladas. Esta operación da origen a combinaciones estructurales típicas que pueden servir para caracterizar y analizar los modelos de gestión y sus variantes, según las combinaciones que pueden surgir de las configuraciones típicas en nada una de las perspectivas. 
En ese predicamento, se puede construir el campo de configuraciones típicas que se articulan en la perspectiva de la regulación, de la siguiente manera:

Tabla 4: Gestión institucional: los procesos de regulación.

Naturaleza de las normas

Reglamentación formal Cultura institucional

Origen de

las normas

Regulación

Externa

Auto

regulación

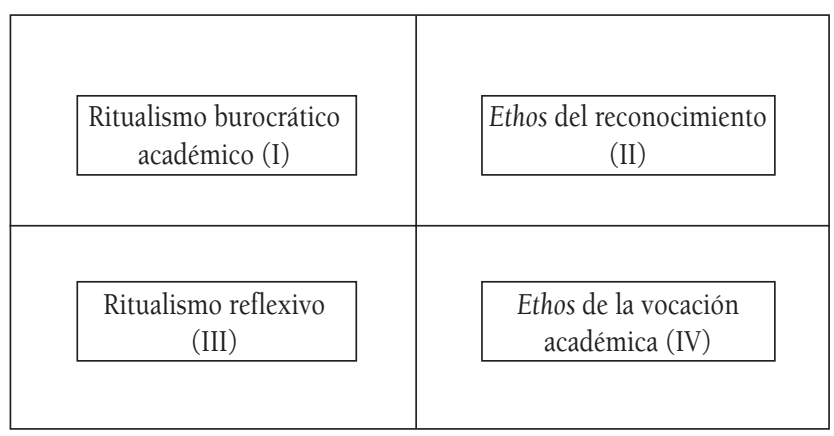

La tabla 4 da origen a cuatro configuraciones normativas (cuadrantes), que se identifican con los números romanos que se inscriben en cada una de ellas. El primer cuadrante resulta de combinar la regulación externa con la regulación formal. En este caso, la regulación externa se refiere a normas de funcionamiento que son dictadas por agentes institucionales del entorno con algún poder de jurisdicción sobre la institución ${ }^{\mathrm{ix}}$. Es más que probable que en esta exteriorización haya varios niveles jerárquicos de normas, en cuyo caso la jerarquía superior indicará normas de aplicación generalizada a todas las instituciones, como son, por ejemplo, las disposiciones de la Ley Orgánica Constitucional de Enseñanza (LOCE), que rige a todas las instituciones de educación superior en Chile.

ix Desde el punto de vista de este análisis no importa, por el momento, si la jurisdicción del agente externo es voluntariamente aceptada por la institución o si es legalmente imperativa. 
Otras normas de niveles jerárquicamente inferiores o subordinados se refieren a grupos de instituciones como son, por ejemplo, las relativas a la distribución del aporte fiscal directo a las universidades del Consejo de Rectores y todavía, en un nivel jerárquicamente más subordinado, las normas aplicables a las universidades católicas que provienen de los organismos pontificios pertinentes. No obstante los diferentes niveles de jerarquía que tocan a la generalidad de su vigencia, todas estas normas tienen en común que provienen del entorno institucional de las universidades y son, por tanto, externas a ellas. El cuadrante (I) agrega a estas normas la condición de que ellas están formalizadas y tienen, por tanto, una expresión explícita y documentada en algún cuerpo claramente identificable destinado a minimizar al rango de interpretación de la norma. Este cuadrante es de tipo legalista y lo hemos denominado como caso representativo del ritualismo burocrático.

Siguiendo en la columna de la reglamentación formal, el cuadrante inferior (III) recoge la combinación de la formalización normativa con las reglas que se originan desde dentro de las instituciones por algún proceso de autorregulación, en cuyo caso habrá que situar las reglamentaciones internas, adoptadas por medio de procedimientos explícitamente convenidos y legitimados, en el seno de la institución. El hecho de que estas normas se originen desde dentro no tiene que ver necesariamente con la rigidez de la forma jurídica que ellas adoptan. Puede haber normas internamente originadas con un alto grado de permanencia estatutaria y rigidez formal del tipo que usualmente asociamos con las normas legislativas externas. Al cuadrante (III) correspondiente lo hemos denominado como ritualismo reflexivo, denotando el carácter autoimpuesto de la norma para distinguirla del rasgo burocrático del otro cuadrante en esta columna en la que se inscriben los dos casos ritualistas.

Pasemos ahora a la segunda columna del cuadro que se despliega bajo la cultura institucional. En el primer cuadrante de esta columna, que hemos identificado con el número (II), se recoge 
la combinación del carácter externo de la norma con el rasgo de estar inscrita en al cultura académica de la institución. Las normas externas susceptibles de esta combinación corresponden a las pautas dictadas por las prácticas de agentes externos que no tienen jurisdicción, pero sí influencia, sobre el comportamiento académico en el interior de las instituciones.

En el caso de las universidades, estas influencias no jurisdiccionales son ejercidas por grupos de referencia que normalmente englobamos bajo términos tales como la comunidad de los científicos, los estándares aceptados por los pares u otra expresión semejante. En este caso, la influencia no jurisdiccional se relaciona con el otorgamiento del reconocimiento, es decir, de la valoración positiva que agentes externos significativos depositan en la institución cuando satisface ciertos criterios.

En suma, se trata de reglas que llevan asociado un alto grado de prestigio concedido por el grupo de referencia respectivo. Por ejemplo, la regla del reconocimiento asociado a la obtención de un grado académico de alta jerarquía, o el prestigio asociado a cierto tipo de publicaciones o el índice de citas que una determinada publicación es capaz de reunir. Todas estas reglas están institucionalizadas en la cultura universitaria, independientemente de que algunas de ellas encuentren una expresión formalizada. Lo destacable aquí es que, estén o no formalizadas estas normas culturales, ellas rigen de verdad la vida académica de las instituciones. Hemos denominado este cuadrante como ethos del reconocimiento.

El cuadrante inferior en la columna de la cultura institucional que se identifica con el número (IV) recoge las normas que se originan desde dentro de la institución por canales que tienen que ver, en mayor o menor grado, con procesos de autorregulación. En general, las reglas de este tipo, vale decir, internamente originadas e inscritas en la cultura institucional, se refieren a prácticas de comportamiento vinculadas con los rasgos distintivos del perfil valórico de la institución y que tienen vigencia en las personas en 
la medida en que éstas se sienten vocacionalmente orientadas o inspiradas por el carácter de la universidad que las acoge. Hay códigos de comportamiento válidos en las universidades estatales o en las universidades católicas que las personas asumen en la medida en que se sienten ellas más realizadas por el carácter de la universidad en la cual trabajan. Hemos denominado este cuadrante como ethos de la vocación académica.

Lo más importante de este análisis es que, desde el punto de vista de las reglas con las cuales se habrá de conducir la gestión institucional, los cuatro cuadrantes (tipos de regulaciones) tienen vigencia simultánea en las universidades, de manera que en cada caso o situación, hay una particular ecuación normativa conforme a la gravitación que tiene en dicha situación cada uno de los tipos de reglas ya descritos, lo cual constituye un factor de alta complejidad para el ejercicio de las funciones de gestión institucional.

Tabla 5: Gestión institucional: las estructuras de gestión.

Legitimación de las decisiones

Participación

Efectividad

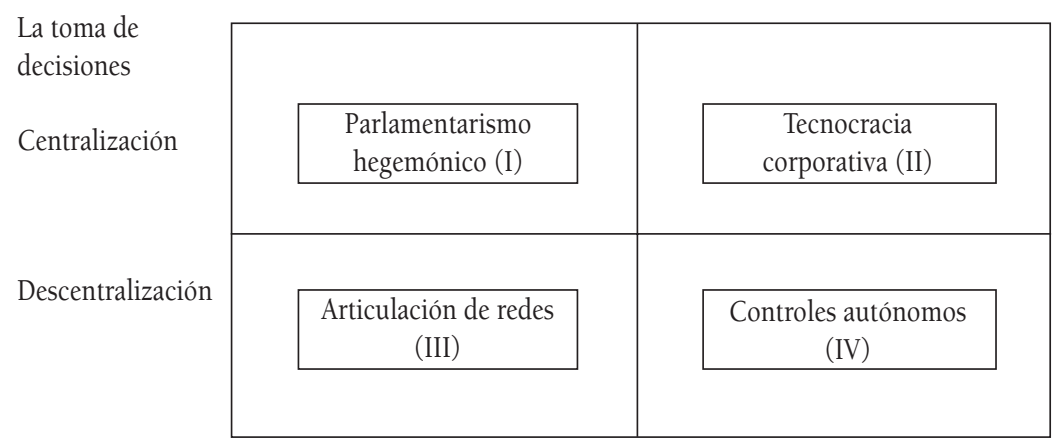

\section{d) Legitimación de las decisiones}

Desde la perspectiva de la legitimación de las decisiones, el tema central está, en mayor o menor grado, centrado en los procesos de participación de los académicos en los asuntos de la universidad, asunto que reviste una doble dimensión: por una parte, la 
circulación de la información, de manera que las personas perciban que están al tanto de lo que pasa en las diversas esferas decisorias de la institución y, por otra, el grado de apertura para la conformación de los grupos y equipos de trabajo, de manera que las personas perciban que sus opiniones son tomadas en cuenta.

La otra dimensión de la participación, que apunta al involucramiento estamental en la toma de decisiones, depende de la conformación y jurisdicción de los cuerpos colegiados de las universidades. Son variados los arreglos estatutarios que regulan la formación y establecen la jurisdicción de estos órganos. En todas las instituciones existe alguna forma de integración de estos cuerpos colegiados por los decanos, vale decir, las autoridades académicas que encabezan agrupaciones de unidades disciplinarias o profesionales afines. Donde sea que estas autoridades estén, el campo de acción del órgano colegiado siempre involucra aspectos que tienen que ver con decisiones académicas en al cúpula de la institución. Éstas se refieren, en general, a políticas transversales de admisión de alumnos, de apertura de carreras con sus planes de estudio y sus grados o títulos, de reclutamiento de personal académico y de reglamentaciones internas.

En estos cuerpos colegiados los decanos representan a las facultades y ellos establecen instancias de deliberación y de coordinación internas en sus respectivas comunidades de profesores, estudiantes y funcionarios. Tienen un doble rol de gestión y de representación. Gestión en el nivel de las facultades y representación en la estructura colegiada superior. Esta doble función se transmite, en cierta forma, al funcionamiento de los órganos colegiados de rango superior (los consejos superiores o sus equivalentes), que son siempre definidos como "autoridades" colegiadas "representativas" para diferenciarlas de las autoridades unipersonales (en esencia, el rector y los miembros ejecutivos de su equipo de gestión).

Es extremadamente interesante lo producido recientemente con los nuevos estatutos de la Universidad de Chile. Vale la pena 
traerlo a colación, aun cuando no forma parte del contenido del informe institucional analizado, pues, a la fecha en que éste se realizó, la universidad estaba recién iniciando el proceso de elaboración de sus nuevos estatutos, que culminó en marzo de 2006.

El punto que interesa aquí es que en esa normativa se distinguen cuerpos colegiados ejecutivos (el Consejo Universitario que equivale a los consejos superiores o juntas directivas, según los matices de cada institución) y cuerpos colegiados representativos (el nuevo "Senado" universitario), cuya función es estrictamente legislativa de tipo general. Los cuerpos colegiados de gestión no tienen canales de participación vinculante (es decir, con derecho a voto) para los estudiantes (que sólo tienen derecho a voz).

El cuerpo colegiado de representación está solamente integrado por representantes elegidos por sus respectivos estamentos, de manera que las instancias de participación que abren el juego a procesos democráticos internos no interfieren con los cuerpos colegiados ejecutivos. Al quedar la conducción ejecutiva de la universidad en manos de los órganos de gestión (uni o pluripersonales), donde estatutariamente no hay representantes del estamento estudiantil con derecho a voto, se salva la prohibición constitucional para esta forma de representación. De paso, con esta diferenciación entre gestión y representación, se limita drásticamente la viabilidad de las estructuras que pudieran darse en el cuadrante del parlamentarismo hegemónico. En el sistema chileno esto simplemente no es factible.

\section{e) La toma de decisiones}

En los informes analizados se observa que las estructuras orgánicas de las instituciones tienden a ser, por lo general, muy centralizadas, lo que se expresa en el muy amplio campo de atribuciones que están depositadas en el rector. La concentración de atribuciones va acompañada, además, por el hecho de que es el rector con su equipo ejecutivo, quien comanda la agenda de funcionamiento efectivo de los órganos pluripersonales superiores. La legitimación de esta centralización descansa en la práctica en los imperativos de 
efectividad que se dirigen a las rectorías en el manejo de los asuntos de financiamiento de las instituciones. La fuerza de estas exigencias de efectividad para la gestión de los recursos económicos, especialmente para el manejo operacional de los flujos financieros, deriva del entorno competitivo donde se encuentran hoy las universidades en el sistema de educación superior chileno, que, por cierto, no excluye a las universidades del Consejo de Rectores.

En los hechos, estas operan en un ambiente complejo de semirregulación, pues, por una parte, acceden por canales fuertemente regulados a una cuota de recursos públicos de manera directa o indirecta (Aporte Fiscal Indirecto, AFI) y a fondos solidarios de ayuda estudiantil que cubren una parte minoritaria de sus necesidades de financiamiento operacional; ellas son cubiertas sustancialmente por los ingresos directos por aranceles de las carreras profesionales y otros programas de docencia.

El artífice de esta operación es siempre el equipo rectorial y, por tanto, el rector. Allí está en el fondo la explicación del alto grado de centralización que tiene la gestión universitaria en Chile. El imperativo de efectividad en la gestión económica empuja naturalmente a la estructura de la gestión hacia el cuadrante de la tecnocracia corporativa, que es percibido como el verdadero nicho donde se adoptan las decisiones institucionales principales.

Las estructuras de gestión no son descentralizadas ni parece que haya una tendencia clara a avanzar en esa dirección. Los cuadrantes de la parte inferior del gráfico son en general espacios vacíos en las instituciones analizadas en este estudio. No se advierte un proceso firme que lleve a la articulación de redes para la gestión institucional ni tampoco se observan desarrollos claros en el establecimiento de controles autónomos en la estructura de la gestión de las universidades.

Hay algunos espacios donde se abre la posibilidad de aplicación de estos controles, como es el caso, por ejemplo, de la implementación 
de procesos de evaluación de la productividad de los académicos, donde las facultades empiezan a insinuar una cierta capacidad autónoma para tal efecto. Lo interesante del caso es que estas experiencias son juzgadas como procesos que ponen en riesgo la sistematicidad de las normas relativas a la carrera académica. En definitiva, en los modelos de gestión de las universidades chilenas hay centralización para mucho rato.

\section{Los temas específicos para el ejercicio de la gestión}

\section{a) La gestión de la información}

La dimensión de la información tiene siempre una doble cara que muestra la complejidad de estos procesos. Una de esas caras es la que se proyecta desde quienes tienen responsabilidad en la difusión de la información, que miran el problema desde el ángulo de los mecanismos y las vías de entrega de datos. En este sentido suele notarse que los responsables de la gestión institucional muestran los modos, las frecuencias y los tiempos de entrega y difusión de información para señalar que desde sus ámbitos de gestión hay una efectiva y generalizada operación de los flujos hacia la comunidad. Todas las universidades manifiestan progresos y logros importantes en el desarrollo de los sistemas informáticos centrales para la recolección y sistematización con el fin de alimentar decisiones de diverso tipo en el nivel central de la institución.

La otra cara es la que se proyecta desde la generalidad de los estamentos académicos, que frecuentemente señalan que la información no se difunde o que se entrega tardíamente y con sesgos. Todo indica que esta discrepancia apunta a una cultura organizacional que no siempre articula en forma adecuada la información formalmente publicitada (mediante boletines, páginas web, circulares y otros medios) por los actores de la gestión institucional y la resonancia efectiva que esa información tiene en los receptores de los estamentos universitarios, que operan con una selección autoinducida de los canales de información que ellos efectivamente usan. Lo probable es que el alcance de los canales formales sea segmentado en función de esta autoinducción. 
En otras palabras, si la información se pone en la página web, ella va a llegar al segmento académico que efectivamente usa la red y navega regularmente en ella. Esto ocurre con todo el resto de los canales formales, de manera que es bastante dudoso suponer que la comunidad académica se va a considerar a sí misma como ampliamente informada de los asuntos relativos a la gestión de la institución por el mero hecho de que aumente la apertura de la información. Esta discrepancia explica el hecho, muchas veces paradojal para los grupos directivos, de que las quejas de desinformación se intensifican con el aumento de los flujos formales de información.

\section{b) La gestión de los recursos humanos}

Del material analizado se puede inferir que las instituciones se esmeran en mostrar medidas orientadas a mejorar la calidad de su recurso humano académico y administrativo, al mismo tiempo que reconocen ciertas dificultades para equilibrar un clima de bienestar y autonomía con las demandas de productividad y rendición de cuentas. La formalización de los cambios en la gestión de recursos humanos puede entrar en tensión con las prácticas arraigadas en la cultura organizacional, pero también con limitaciones materiales que aumentan la sensación de conflicto entre "lo que se acata" y "lo que lo que cumple", entre "lo que se quiere" y "lo que se puede", entre "lo que se dice" y "lo que se hace".

En la mayoría de las instituciones se busca privilegiar el reclutamiento de académicos jóvenes y con posgrado, asunto que, sin embargo, presenta diversas dificultades para su adecuada implementación. Estas dificultades se asocian a veces con las cortapisas derivadas de las distintas normativas de contratación y jerarquización que rigen dentro de las facultades o con la falta de una clara política de inducción que complemente el sistema de selección.

Un problema recurrente es el que se refiere al insuficiente seguimiento del desempeño, que afecta incluso a los casos donde el proceso de selección es percibido como adecuado. Una de las 
formas como tiende a controlarse el rendimiento académico es por medio de los complementos de asignaciones por productividad académica. En las instituciones que están operando en esta dirección suele haber dificultades para el control jerárquico efectivo de los académicos, directivos y administrativos. Ellas impiden una apropiada medición de la productividad y, por tanto, afectan la aplicación y la transparencia de tales asignaciones. Los estudios de posgrado entre los académicos no necesariamente facilitan el sostenimiento de la productividad.

Hay, por otra parte, instituciones en las cuales no se aplica aún una evaluación periódica de productividad ni se la asocia a incentivos. No obstante, todas las instituciones están aplicando formas de evaluación del desempeño de la docencia, pero con distintos grados de efectividad. En algunos casos se reconoce que hay insuficiencias en el liderazgo académico y administrativo para realizar la evaluación de este desempeño. También es generalizada la aplicación de instrumentos de evaluación de los docentes por parte de los estudiantes, pero no es posible discernir un patrón sistemático de acciones respecto de los docentes que son mal evaluados.

Se reconoce la importancia de la capacitación y se entregan antecedentes sobre la forma como se llevan a cabo iniciativas para este fin. Sin embargo, se observa una variedad de problemas que afectan estas acciones como, por ejemplo, la falta de políticas sistemáticas sobre el tema y las limitaciones que encuentran algunas instituciones para acogerse a beneficios que subsidian estos programas (Servicio Nacional de Capacitación y Empleo, SENCE). Algunas instituciones indican que el apoyo al perfeccionamiento termina "subsidiando" a universidades de la competencia, toda vez que se invierte en capacitación y el profesor sigue prestando servicios en otra institución.

En la mayoría de los casos existen normativas y comisiones que reglan los nombramientos y la carrera académica. En otros casos, sin embargo, las normas concernientes a la evaluación 
resultan rígidas y poco funcionales o se indica que hay marcadas diferencias en las normativas de calificación académica entre facultades de la misma institución. Algunas organizaciones han buscado aplicar mecanismos (incorporación de académicos externos a las comisiones que deciden sobre calificación) con el objetivo de aumentar la transparencia de estos procesos. Casi todas las universidades tienen problemas con la renovación de sus cuadros académicos, en buena medida ocasionados por la insuficiencia de los incentivos a la desvinculación, o por la liquidación de una pensión tope que no es atractiva. En general, se diagnostican problemas serios para motivar que los académicos, que no forman parte de equipos consolidados, desarrollen actividades regulares de investigación, y cuando lo hacen la difusión de su investigación suele ser deficiente.

Las instituciones reconocen que la existencia de cortapisas burocráticas y normativas rígidas atenta contra el desarrollo de una cultura orientada a la calidad de los desempeños. Este problema sería no sólo de orden administrativo, sino también de cultura organizacional y afecta la productividad docente y de investigación. En esa lógica, las instituciones tienen altas expectativas en la adopción de algún sistema de incentivos asociados a remuneraciones, así como en la evaluación sistemática de los académicos en todas las dimensiones que componen su quehacer, pero persiste la duda acerca de cuántas de las trabas observadas son modificables mediantes estatutos y cuántas a través un manejo más hábil de los factores culturales involucrados.

\section{c) El crecimiento expansivo y sedes}

En relación con el tema del crecimiento vinculado a la apertura de sedes, es claro que en este grupo del primer ciclo de acreditación son pocas las instituciones que han adoptado esta modalidad de expansión. Esto en marcado contraste con muchas de las instituciones que se anotaron en los posteriores ciclos de acreditación. 
Dos factores están notoriamente presentes en este comportamiento institucional mesurado. Por una parte, las universidades situadas en la Región Metropolitana tienen una importante ventaja comparativa, en cuanto ellas están en el espacio apropiado para captar los importantes volúmenes de población estudiantil universitaria potencial que siguen concentrados en esta región. Una universidad que no esté en Santiago, o sus zonas directas de influencia, difícilmente podrá aspirar a tener una captación significativa de matrícula, debido simplemente a la enorme densidad de población de potenciales alumnos que existe allí en comparación con cualquier otra localización (con la posible excepción de la zona de Concepción).

En este marco, no hay en verdad muchos incentivos para las universidades de la Región Metropolitana, en el sentido de abrazar una política de apertura de sedes. Aparte de estas universidades, en el grupo estudiado hay un importante conjunto de instituciones no estatales que tienen una aproximación más firme respecto de la misión que están llamadas a cumplir en cuanto su diseño original o fundacional. El crecimiento en estas instituciones tiende a mantenerse dentro de su localización central, con lo cual no se ha producido en ellas la dinámica que empuja a la apertura de sedes, donde reconocidamente se dificulta la mantención permanencia y el aseguramiento de la calidad de los programas ofrecidos.

Con todo, en el grupo analizado hay experiencias limitadas de este proceso de crecimiento expansivo mediante la apertura de sedes, y ellas corresponden a instituciones de carácter regional que han enfatizado en su gestión institucional el constreñimiento real (actual y proyectado) de la matrícula en la región correspondiente. Esto no quiere decir que todas las universidades del grupo estudiado que están situadas en regiones adopten este tipo de estrategia expansiva; pero, cuando ella se produce, la institución es de las que están en regiones. 


\section{d) La gestión económica}

Todas las instituciones de este grupo destacan su fuerte dependencia de los ingresos mediante aranceles para su financiamiento. En la combinación de sus fuentes de financiamiento -aranceles, Aporte Fiscal Directo (AFD), Aporte Fiscal Indirecto (AFI), fondos de desarrollo institucional (FDI), aportes de los fondos solidarios de ayuda estudiantil, proyectos concursados a fondos competitivos (Programa de Mejoramiento de la Calidad y Equidad de la Educación Superior, MECESUP), venta de servicios y, eventualmente, donaciones- el ingreso por aranceles es el de mayor magnitud y uno de los más predecibles (excepto el AFD, que tiene fijación histórica, pero es considerablemente exiguo, especialmente para las universidades que se derivaron de la Pontificia Universidad Católica de Chile, o de la Universidad de Chile y la Universidad de Santiago de Chile, combinadas).

Esta circunstancia ha favorecido una cuidadosa gestión del proceso de admisión y matrícula y una especial dedicación a evitar o minimizar la deserción estudiantil. Muchas instituciones han llegado incluso a calcular el monto de ingreso marginal que representa la entrada de un alumno. Este es un efecto favorable en el sentido de promover una gestión económica de estos recursos que es cada vez más eficiente. Sin embargo, es preciso notar que esta fuente de ingreso sustenta básicamente el financiamiento operacional de las instituciones.

El aspecto deficitario en este rubro es el manejo y el control eficaz de la morosidad. La gestión que hacen las instituciones de sus carteras de alumnos morosos es notoriamente ineficiente, con algunas excepciones muy precisas. Hay que reconocer, por cierto, que este es un tema de altísima sensibilidad política y que, en el grupo de universidades públicas que se han analizado, afecta y complica seriamente las relaciones de la universidad con las organizaciones estudiantiles. Hay, por tanto, una evidente racionalidad "política" que explica la renuencia de los actores de la gestión institucional a sumir conductas financieramente más eficaces de recaudación de aranceles morosos. 
Otra cosa es el financiamiento de la inversión, donde el cuadro es mucho más variado y más errático. El punto más importante en este rubro es el del endeudamiento en el sistema financiero, que suele provenir de las aportaciones de contrapartida que las instituciones tienen que cubrir para acceder a los fondos de desarrollo (sean estos concursables o no).

La mayoría de las instituciones acusa serios problemas para constituir y gestionar fondos de retiro que posibiliten la desvinculación programada de personal para inducir la renovación de los recursos humanos, especialmente de los académicos. Salvo excepciones, esta debilidad de la gestión económica induce a que las instituciones no adopten iniciativas tendientes a generar retiros y jubilaciones a las edades en que ello es posible. Hay aquí una inercia institucional que favorece a las dos partes involucradas; a la universidad que carece de fondos de desahucio en la magnitud necesaria y a los académicos que prefieren continuar laboralmente activos, porque los montos exiguos de la jubilación mensual representan una intolerable disminución de sus ingresos.

En síntesis, se puede observar que la gestión de la economía institucional está marcada por una administración financiera operacional eficiente, un control estricto de los gastos en insumos, una actitud tolerante hacia la alta morosidad, un manejo controlado del endeudamiento financiero y una inercia consensuada en la gestión de los fondos de retiro. El conjunto de estos predicamentos de la gestión económica es ampliamente funcional a un manejo ejecutivo y administrativo altamente centralizado, que se deposita en las vicerrectorías que se hacen cargo de estas tareas.

\section{e) La gestión de los programas}

Todas las instituciones de este grupo están involucradas, en mayor o menor grado, en los temas de la innovación del proceso formativo de sus estudiantes. Es destacable que en todas ellas se haya producido un notorio giro de lenguaje, en el sentido de empezar a manejar conceptos para significar una educación centrada en el 
aprendizaje de los alumnos más que en la enseñanza magistral. Esto supone la introducción de métodos innovadores que favorecen un aprendizaje activo por parte de los estudiantes, entre los cuales se privilegia de especial manera el uso de medios audiovisuales y el desarrollo de infraestructura multimedial. Más allá de estas innovaciones de discurso y de materialidad de la docencia, hay varias instituciones que han empezado a abordar el tema más sustantivo de la innovación curricular.

Destaca el hecho de que, en la mayoría de los casos, este proceso está empezando a cambiar el papel y la configuración de las vicerrectorías académicas, las que, sin dejar de ser las instancias de coordinación general de la docencia, empiezan a asumir una función de unidades gestionadoras de la innovación. Aquí hay un fenómeno importante e interesante de analizar y de evaluar, pues todo indica que en este giro se está incubando un proceso de cambio de gran envergadura en las universidades. La evaluación de la productividad de los docentes, en cuanto tales, y del rendimiento de los estudiantes sigue siendo un problema no resuelto todavía en plenitud, pero eso no aminora el potencial de cambio que está produciéndose con este giro en la función de las vicerrectorías académicas.

\section{Conclusiones}

Como se ha visto, la articulación de las áreas de problemas para la gestión institucional se logra por medio del establecimiento de algunas polaridades transversales, que pueden ser útiles para modelar patrones de gestión institucional donde se pueden ordenar y hacer relativamente coherentes los tópicos que se han identificado anteriormente.

La primera de estas polaridades transversales se construye en términos de un eje que se extiende desde el polo de la participación ampliada hasta el polo de la efectividad. Participación ampliada quiere decir aquí una especie de apertura a procesos de involucramiento 
proactivo de los académicos y de los distintos miembros de la comunidad universitaria. Esta participación contrasta con la efectividad, en una combinación que es compleja, pero posible. En efecto, la articulación de participación con efectividad supone incursionar en el segundo eje que se extiende desde el polo de la centralización hasta el de la descentralización, considerados como principios de construcción de la arquitectura de la gestión.

Un tercer eje apunta a la naturaleza de la normatividad institucional. Se extiende desde el polo de la de racionalización formal, normativa reglamentaria propia de instituciones que son muy proclives a disponer de una gama muy detallada de información, manuales de procedimientos, reglamentos, instancias de interpretación de los reglamentos, hasta el polo de la cultura institucional de la universidad, donde la normatividad se construye más por prácticas y hábitos colectivamente aceptados como válidos, que por reglamentación normativa. Finalmente, se puede discernir un cuarto eje que se extiende desde el polo de los procesos de control inducidos externamente hasta el de los autorregulados.

Para la caracterización de los modelos de gestión pueden identificarse dos campos principales. El primero, el campo de la participación, donde se pueden combinar dos de los cuatro ejes, a saber: descentralización-centralización y participación-efectividad. El segundo campo es el de la regulación, donde se pueden articular el eje del control externo-autorregulación con el eje de la regulación normativa formal-cultura institucional. El cruce de estos dos campos determina los cuadrantes donde se puede ubicar el núcleo decisivo del "modelo de gestión" correspondiente.

En general, si se considera la matriz de estructuras de gestión (tabla 5), se observa que las instituciones tienden a poner en práctica modelos de gestión centrados en el cuadrante de las tecnocracias corporativas con una participación restringida que impide el desarrollo de un parlamentarismo más o menos generalizado (hegemónico). Este cuadrante está funcionalmente apoyado en una 
fuerte centralización de la toma de decisiones con escasa articulación de redes y exiguos controles internamente autónomos. Si se atiende al complejo de sus normas y regulaciones, los modelos de gestión son más variados y es posible observar todos los cuadrantes de la respectiva matriz (tabla 4).

Sea cual sea el modelo de gestión que se materialice en las instituciones, es indispensable tomar nota de las características del contexto sistémico en el cual se desenvuelve la acción de las autoridades de las universidades del Consejo de Rectores y, dentro de ellas, de las universidades estatales. Considerando los principales rasgos del perfil que ha venido tomando el sistema de educación superior en Chile a lo largo de las últimas décadas, el escenario sistémico indicado apunta a los siguientes aspectos centrales:

- Como principio general, el modelo de universidad pública que se requiere es muy distinto del de los 60 y de principios de los 70. La economía de ese entonces crecía hacia adentro y las empresas no tenían competencia entre sí o tenían un mercado muy asegurado. Ahora la economía nacional se desenvuelve en un contexto distinto, con procesos de producción y servicios globalizados, y con fuertes exigencias de competitividad para las empresas, lo que las obliga a interactuar con la universidad.

- Un tema crítico es lograr un nivel de financiamiento mínimo asegurado para las universidades estatales, política que puede ser extensible al resto de las universidades pertenecientes al Consejo de Rectores. Para su determinación se tiene que considerar la dimensión social de la educación superior estatal, elemento de equidad que es consustancial a la misión de la universidad pública.

- Diseño y aplicación de una clara política de bienestar, que permita abordar de manera efectiva los problemas de permanencia de los alumnos en el sistema, que vaya más allá de los mecanismos de financiamiento de los costos de aranceles que están operando por medio de créditos o becas según el caso. 
- Autonomía responsable de la gestión universitaria, que permita el aseguramiento de la calidad de los procesos y resultados de las instituciones, y que posibilite la operación de mecanismos para hacer exigible la responsabilidad por los recursos públicos que se reciban, en particular, la oferta de carreras profesionales que debiera regirse por alguna forma de contrato de desempeño.

- Desarrollo y aseguramiento de la capacidad de las universidades públicas para responder a los requerimientos de la economía nacional, que se sustentan en el aprovechamiento de la competitividad del país. Este debe orientar ahora su crecimiento y desarrollo económico hacia los mercados externos.

- Instalar en las universidades estatales una estructura organizacional más eficiente, que haga más horizontales las jerarquías burocráticas, para que las cadenas de decisión sean menores y la gestión gane en agilidad. Para normar la participación en estas estructuras organizacionales, es preciso distinguir -en lo que se refiere a la administración global de la universidad-las instancias en las cuales se discutan o se perfilen las tomas de decisiones que van a afectar a las instituciones en el largo plazo, de aquellas en las cuales se genera la discusión, regulación, armonización y la solución de problemas de corto plazo. 
ANEXO

Matriz de codificación de informes institucionales

\begin{tabular}{|c|c|c|c|c|}
\hline & Objetivos & $\begin{array}{l}\text { Instrumentos/ } \\
\text { estructuras } \\
\text { actuales y en } \\
\text { desarrollo }\end{array}$ & $\begin{array}{l}\text { Dificultades } \\
\text { detectadas } \\
\text { (universidades y } \\
\text { CNAP) }\end{array}$ & $\begin{array}{l}\text { Lógica de gestión } \\
\text { que emerge y/o } \\
\text { ejes en tensión }\end{array}$ \\
\hline $\begin{array}{l}\text { Cautela de la } \\
\text { misión }\end{array}$ & - & & & \\
\hline $\begin{array}{l}\text { Estrategia de } \\
\text { desarrollo }\end{array}$ & $\bullet$ & & $\bullet$ & • \\
\hline $\begin{array}{l}\text { Estructura } \\
\text { organizacional }\end{array}$ & & • & & \\
\hline Gestión económica & $\bullet$ & & • & \\
\hline $\begin{array}{l}\text { Gestión de la } \\
\text { información }\end{array}$ & & • & & • \\
\hline $\begin{array}{l}\text { Gestión de recursos } \\
\text { humanos }\end{array}$ & $\bullet$ & & - & \\
\hline $\begin{array}{l}\text { Crecimiento } \\
\text { cuantitativo }\end{array}$ & $\bullet$ & & & \\
\hline $\begin{array}{l}\text { Gestión de las } \\
\text { carreras }\end{array}$ & $\bullet$ & & • & \\
\hline $\begin{array}{l}\text { Gestión de las sedes } \\
\text { e infraestructura }\end{array}$ & & • & & • \\
\hline $\begin{array}{l}\text { Gestión de } \\
\text { investigación y } \\
\text { vinculación con } \\
\text { el medio }\end{array}$ & & & (sólo en CNAP) & \\
\hline
\end{tabular}

Recibido: 26 de mayo de 2006

Aceptado: 10 de junio de 2006 